\title{
ELECTRONIC REGISTRY SYSTEM FOR DIABETIC PATIENTS UNDERGOING HYPERBARIC OXYGEN THERAPY: DETERMINATION OF COMMON DATA ELEMENTS
}

\author{
Shahram Oliaei ${ }^{\mathrm{a}}$, Nahid Mehrabi ${ }^{\mathrm{b}}$, Tayebeh Nooric ${ }^{\mathrm{c}}$, Solmaz Saeidi ${ }^{\mathrm{d}}$ and Esmaeil Mehraeen ${ }^{\mathrm{e}, 1}$ \\ ${ }^{a}$ HBOT Research Center, Golestan Hospital, Islamic Republic of Iran, Navy and AJA Medical University, Tehran, Iran \\ ${ }^{b}$ Assistant Professor of Health Information Management, Aja University of Medical Sciences, Tehran, Iran \\ ${ }^{c}$ Department of Health Information Technology, School of Allied Medical Sciences, Zabol University of Medical \\ Sciences, Zabol, Iran \\ ${ }^{d}$ Department of Nursing, Khalkhal University of Medical Sciences; Khalkhal, Iran \\ ${ }^{e}$ Department of Health Information Technologies, Khalkhal University of Medical Sciences, Khalkhal, Iran
}

\begin{abstract}
Background: The advent of electronic registries has increasingly provided an opportunity to improve healthcare services and advanced applied research. However, the delivery of effective hyperbaric technology services requires well-functioning electronic registry systems and patient information integration to support national and global analyses. Objectives: this study aimed to determine the most common data elements of an electronic registry for diabetic patients undergoing hyperbaric oxygen therapy (HBOT).

Methods: This was a cross-sectional study that conducted in two main stages. The first phase involves a review of the current available evidence on the study aim. In this stage, we searched related articles in PubMed, Web of Science, Up To Date, Science Direct, and Scopus databases. In the next phase, using the researcher-made questionnaire, the essential data elements and technical features of an electronic registry system were validated. The statistical population of the present study consisted of 32 endocrinologists working in Golestan Hospital. The collected data was analyzed using descriptive statistics in SPSS version 26.

Results: Through a full-text review of 12 related articles, the data elements $(\mathrm{N}=95)$ of an electronic registry for diabetic patients undergoing HBOT were identified and classified into three categories including: administrative $(\mathrm{n}=30)$, clinical $(\mathrm{n}=54)$, and technical $(\mathrm{n}=11)$. According to findings, among the 30 data elements of administrative category, 25 data elements and in clinical category, 50 data elements were selected by statistical population. Moreover, all identified technical features were selected by experts, except the "decision making support".

Conclusions: In this study we identified and validated the data elements and technical features of an electronic registry system for HBOT-treated diabetic patients. These requirements make it possible to standardize data collection and improve health care services.
\end{abstract}

\section{KEYWORDS}

Data Elements, Diabetes, Registry System, Hyperbaric Oxygen Therapy, Diving Medicine

\section{INTRODUCTION}

Diabetes mellitus is one of the culprits of public health problems that have led to considerably widespread economic and social consequences worldwide due to its high prevalence (Irawan et al., 2018). Complications can include kidney failure, diabetic foot ulcers, and even lower limb amputation. Oxygen plays an important role in wound healing. However, the main components of standard treatment are glycemic control, antibiotic medication, wound debridement (depletion of dead tissues), wound care, offloading and improvement of blood flow / revascularization (Saputri et al., 2018).

\footnotetext{
${ }^{1}$ Corresponding Author: Esmaeil Mehraeen, Assistant Professor, Department of Health Information Technology, Khalkhal University of Medical Sciences, Khalkhal, Iran. Postal code:5681761351, tel:+98-4532426801, fax: +98-4532422305,
}

Email: es.mehraeen@gmail.com 
Hyperbaric Oxygen Therapy (HBOT) causes $100 \%$ breathing of oxygen under increasing atmospheric pressure conditions. HBOT involves breathing of pure oxygen into a room or enclosure that is used in a variety of medical settings (Hunt, 1972). During high pressure oxygen therapy, the blood hemoglobin in the vein is completely saturated with oxygen and results in increased oxygen pressure in the blood vessels (Hunt, 1972, Ruthenborg et al., 2014). HBOT treats several medical conditions such as severe infections, air bubbles in the blood vessels, and wounds caused by diabetes. Standard care for diabetes includes condition control; diabetes wound care and infection treatment. Diabetic foot is the most commonly diagnosed condition for HBOT control in diabetics. In addition to reducing insulin requirement in diabetic patients, other factors such as decreased oxygenation (hypoxia), edema, and HBOT-induced infection are reduced (Fife and Eckert, 2018).

Hyperbaric specialists are widely monitored for compliance with health insurance coverage policies and adherence to clinical practice guidelines. The best way to achieve this is registering clinical information; clinical data registration is nowadays performed using computerized registry systems (Mathieu et al., 2017). Registry systems are currently available for a wide range of clinical conditions such as infectious diseases, cancers, congenital diseases and rare diseases such as cystic fibrosis. Chronic diseases such as AIDS, diabetes, heart failure and kidney disease have been the target of registry systems in many countries in the final stages (Al-Rubeaan et al., 2013). A survey in 1040 physician organizations showed that diabetes registries were used in $40.3 \%$, asthma registries in $31.2 \%$, congestive heart failure registries in $34.8 \%$, and depression registrations in $15.7 \%$ of health care organizations (Casalino et al., 2003).

In recent years, the creation of registry systems in this area is essential for data management due to the increase in oxygen hyperbaric services (Andersen et al., 2016, Noori et al., 2019). Patients undergoing HBOT treatment should have their information recorded in a system known as an electronic data recording system (such as a HBOT-treated wound information system). The data recorded in these systems may be used for benchmarking, service quality reporting, health policy or specialized data projects. Data may be requested for criteria, quality reporting, public policies or specialized data projects. The Wound Information Record System can provide benchmarking information for many wound care interventions and blood glucose medications by using the Health Record documentation for each HBOT-treated patient. This will provide the infrastructure needed to implement similar technologies such as Electronic Health Record (EHR) and distant patient information access (Fife and Eckert, 2018).

One of the key components of health registry systems is availability of standard data elements for proper collection and timely access to health information (Andersen et al., 2016). Therefore, gathering demographic and clinical data using standard data elements can be useful in providing good health care to patients with hyperbaric diabetes (Hosseini et al., 2010). In other words, standard data elements will avail detailed data to service providers. Standard and accurate data require access to valid patient information, infections, new therapies, and their impact (Ahmadi et al., 2014).

An electronic registry with standard data elements is significantly needed to enable accurate data collection in developing countries as HBOT progresses (Fife et al., 2016). There are few studies on the data elements of the registry system for HBOT-treated diabetic patients. As a result, unclear and incomprehensible data are used in HBOT-based care services (George et al., 2017). Hence, if we lack standard data elements to collect and access accurate and valid data, it may be impossible to pot for an empirical approach to improve the delivery of health services, monitoring and ongoing evaluation of diabetic patients at HBOT centers. This study aims to determine common data elements of a registry system for diabetic patients treated with HBOT.

\section{MATERIALS AND METHODS}

This was a cross-sectional study that conducted in two main stages. The first phase involves a review of the current available evidence to determine the data elements of an electronic registry system for diabetic patients undergoing hyperbaric oxygen therapy. In this stage, we searched related articles in PubMed, Web of Science, Up To Date, Science Direct, and Scopus databases. Hybrid keywords including hyperbaric oxygen therapy, HBOT, electronic registry, e-registry, registry system, information system, database, diabetes, technical functionality/features, data element and minimum data set were used to come up with the search strategy. 
The inclusion criteria included full text of English-written original or review papers which had been published from 2010 to 2020. Abstracts, reports, letters to the editor, and news articles were excluded from the study. First, the title and abstract of articles were evaluated according to inclusion and exclusion criteria. The full text of selected articles was then studied for further evaluation of final selection.

To evaluate the quality of the final selected articles, a checklist containing 15 items (Table 1) was developed based on the related studies (BinDhim et al., 2015, Zapata et al., 2015, McKay et al., 2018). The quality of articles was then rated on three scales: low quality (0-5), medium quality (6-10) and high quality (11-15).

Table 1. Quality assessment checklist

\begin{tabular}{c|l}
\hline NO. & Question \\
\hline 1 & Does the study address any research question(s) or objective(s)? \\
2 & Does the study provide any theoretical framework for the evaluation method? \\
3 & Does the theoretical framework of the study include any health promotion theory? \\
4 & Does the study provide a timeframe of the data collection? \\
5 & Does the study identify the country where the search was conducted? \\
7 & Does the study mention that the reviewed registry system (RS) was downloaded for evaluation? \\
8 & Does the study discuss the selection criteria for HBOT to be included or excluded for review? \\
9 & Does the study provide a clear description of the evaluation method? \\
10 & Is a there at least two independent data extractors with a consensus procedure in place in case of disagreement? \\
11 & Does the study discuss the findings of the evaluation? \\
12 & Does the study look at the reviewed HBOT registry system to promote healthcare services? \\
13 & Does the study discuss any limitations? \\
14 & Does the study provide any future recommendations in general? \\
15 & Does the study state any conflict of interest? \\
\hline
\end{tabular}

In the second phase, a questionnaire containing the identified data elements was designed. The questionnaire was completed within ten days first by four health information management specialists; then, four endocrinologists examined it to ensure its reliability. The collected data were analyzed by SPSS software version 26, and the reliability of the questionnaire was calculated through Cronbach's alpha $(=0.87)$. Eight experts evaluated the content validity of the questionnaire. In order to increase the number of participants in the study, in-person referral method was used.

Finally, a researcher-made questionnaire was administered to the statistical population. The statistical population of the present study consisted of 32 endocrinologists working in Golestan Hospital (The only center equipped with HBOT services in Iran). To analyze the data, the questionnaire items ranged from 1 to 3 (1 for disagreement, 2 for no idea and 3 for agreement). The collected data were analyzed through SPSS software using descriptive statistics (frequency distribution and mean reports). Each data element with a mean score of at least 1.5 and more was selected as the data element of the electronic registry system for diabetic patients treated with HBOT.

\section{RESULTS}

Using the aforementioned strategies, 980 sources were retrieved.

\subsection{General Specifications}

After reviewing the titles and abstracts of the articles, 370 duplicates were found. The 610 titles of the remaining articles were reviewed and then 91 studies with the main selection criteria were extracted. After reviewing the full text of the articles, 12 articles met the study selection criteria and were included in the final review (Figure 1). These 12 studies were published between 2011 and 2019. The mean quality score of the selected articles reviewed was 13 (in the range of 11 to 15 ) indicating high quality of the articles. 


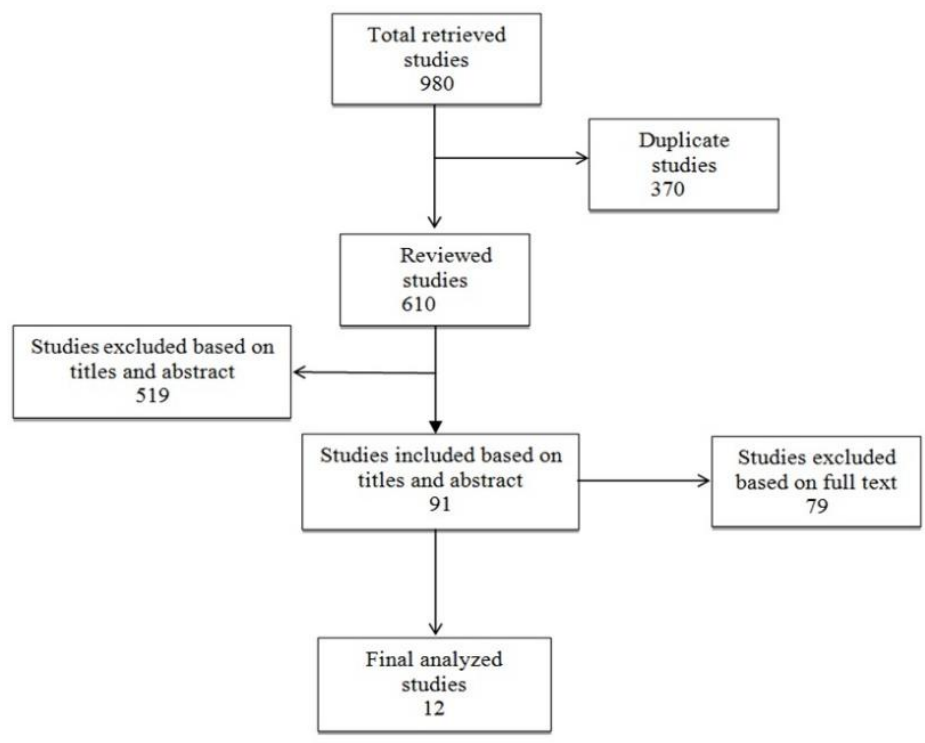

Figure 1. Flow diagram of selection process

\subsection{Evaluating Classifications}

In this study, a set of data elements and features $(\mathrm{N}=95)$ of the electronic registry system for HBOT diabetic patients were identified in three categories: administrative data elements, clinical data elements and technical features. Table 2 shows each of the classifications associated with the reviewed articles. Based on the findings, more than half of the studies (seven studies) described clinical data elements and five studies investigated the technical features of the registry system related to diabetic patients who had been treated with HBOT.

Table 2. Identified categories of data elements and features and final surveyed articles

\begin{tabular}{l|ccc}
\hline \multirow{2}{*}{ Reference } & \multicolumn{2}{|c}{ Data Elements/Capabilities } \\
\cline { 2 - 3 } & Administrative & Clinical & Technical \\
\hline (Sundh et al., 2019) & & & $\sqrt{ }$ \\
\hline (Khorasani et al., 2019) & $\sqrt{ }$ & \\
\hline (Kawecki et al., 2018) & & $\sqrt{ }$ \\
\hline (Fife and Eckert, 2018) & & $\sqrt{ }$ \\
\hline (Irawan et al., 2018) & $\sqrt{ }$ \\
\hline (Lakshminarayanan et al., & $\sqrt{ }$ \\
2017) & & $\sqrt{ }$ \\
\hline (George et al., 2017) & & $\sqrt{ }$ \\
\hline (Fife et al., 2016) & $\sqrt{ }$ \\
\hline (Andersen et al., 2016) & & $\sqrt{ }$ \\
\hline (Stoekenbroek et al., 2014) & & $\sqrt{ }$ \\
\hline (Al-Rubeaan et al., 2013) & & $\sqrt{ }$ \\
\hline (Richesson, 2011) & & \\
\hline
\end{tabular}

\subsection{Sub-Categories}

According to the findings, the administrative category consisted of five subcategories and 30 data elements, the clinical category consisted of six subcategories and 54 data elements, and the technical category consisted of one subcategory and 11 features. The identified data elements and features were scored by 30 samples of attended specialists (response rate was 93.8\%). The results showed that among the 30 data elements of administrative category, 25 data elements and in clinical category, 50 data elements were selected by statistical population (Table 3 and 4). Moreover, all technical features except "decision making support" were selected by the experts (Table 5). 
Table 3. The mean of given scores and selected administrative data elements

\begin{tabular}{|c|c|c|c|c|c|}
\hline Category & Subcategory & No & Data element & Mean & $\sqrt{/} *$ \\
\hline \multirow{30}{*}{ 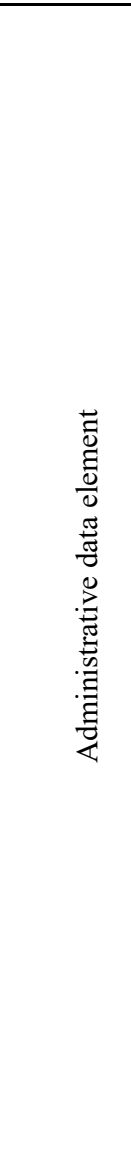 } & \multirow{16}{*}{ Demographic } & 1 & Patient medical record number & 3 & $\sqrt{ }$ \\
\hline & & 2 & National identity number & 3 & $\sqrt{ }$ \\
\hline & & 3 & Patient name & 3 & $\sqrt{ }$ \\
\hline & & 4 & Patient surname & 3 & $\sqrt{ }$ \\
\hline & & 5 & Father name & 2.8 & $\sqrt{ }$ \\
\hline & & 6 & Sex & 2.9 & $\sqrt{ }$ \\
\hline & & 7 & Date of birth & 3 & $\sqrt{ }$ \\
\hline & & 8 & Country of birth & 2.9 & $\sqrt{ }$ \\
\hline & & 9 & Province of birth & 2.5 & $\sqrt{ }$ \\
\hline & & 10 & City of birth & 2.9 & $\sqrt{ }$ \\
\hline & & 11 & Occupation & 2.1 & $\sqrt{ }$ \\
\hline & & 12 & Marital status & 1.3 & * \\
\hline & & 13 & Education level & 2 & $\sqrt{ }$ \\
\hline & & 14 & Economic status & 1 & * \\
\hline & & 15 & Patient's address & 2.5 & $\sqrt{ }$ \\
\hline & & 16 & Phone/mobile number & 2.4 & $\sqrt{ }$ \\
\hline & \multirow{5}{*}{ Patient encounter } & 17 & Date of admission or referral to the facility & 3 & $\sqrt{ }$ \\
\hline & & 18 & Time of admission or referral to the facility & 2.1 & $\sqrt{ }$ \\
\hline & & 19 & Name of institution or referral center & 2.9 & $\sqrt{ }$ \\
\hline & & 20 & Name of referrer person/ physician & 2.5 & $\sqrt{ }$ \\
\hline & & 21 & Informed consent & 3 & $\sqrt{ }$ \\
\hline & \multirow{3}{*}{ Provider ID } & 22 & Name of the service provider person & 1.1 & * \\
\hline & & 23 & Family name of the service provider person & 1.2 & $\sqrt{ }$ \\
\hline & & 24 & Identifier code of the service provider person & 2 & $\sqrt{ }$ \\
\hline & \multirow{4}{*}{ Facility identifier } & 25 & Facility Name & 2.3 & $\sqrt{ }$ \\
\hline & & 26 & Identification code of the facility & 2.1 & $\sqrt{ }$ \\
\hline & & 27 & Full facility address & 1.4 & * \\
\hline & & 28 & Telephone of facility & 1.3 & * \\
\hline & \multirow{2}{*}{ Financial data } & 29 & Primary insurer company & 2 & $\sqrt{ }$ \\
\hline & & 30 & Insurance number & 3 & $\sqrt{ }$ \\
\hline
\end{tabular}

$\sqrt{ }=$ Selected data element; $*=$ Removed data element

Table 4. The mean of given scores and selected Clinical data elements

\begin{tabular}{|c|c|c|c|c|c|}
\hline Category & Subcategory & $N$ & Data element & Average & $\sqrt{/} *$ \\
\hline \multirow{19}{*}{ 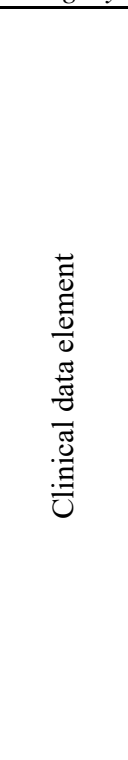 } & \multirow{2}{*}{ Patient CC } & 1 & Chief Complaint of patient/referral & 2.7 & $\sqrt{ }$ \\
\hline & & 2 & Current condition/disease sign & 3 & $\sqrt{ }$ \\
\hline & \multirow{13}{*}{$\begin{array}{l}\text { Personal medical } \\
\text { history }\end{array}$} & 3 & Diabetes type & 3 & $\sqrt{ }$ \\
\hline & & 4 & Diabetes duration & 2.5 & $\sqrt{ }$ \\
\hline & & 5 & Diabetic retinopathy & 3 & $\sqrt{ }$ \\
\hline & & 6 & Family history of diabetes & 2.9 & $\sqrt{ }$ \\
\hline & & 7 & Chronic Disease and Comorbidity & 2.6 & $\sqrt{ }$ \\
\hline & & 8 & Alcohol consumption/addiction-Opium abuse/addiction & 2.8 & $\sqrt{ }$ \\
\hline & & 9 & Type of opium & 2.1 & $\sqrt{ }$ \\
\hline & & 10 & The name of the medications used & 2.6 & $\sqrt{ }$ \\
\hline & & 11 & Name of the allergicsubstance or drug & 2.7 & $\sqrt{ }$ \\
\hline & & 12 & Description of specific nutritional habits & 3 & $\sqrt{ }$ \\
\hline & & 13 & Surgical procedure history & 2.5 & $\sqrt{ }$ \\
\hline & & 14 & Surgical procedure & 1.3 & * \\
\hline & & 15 & surgical procedure names & 1.9 & $\sqrt{ }$ \\
\hline & \multirow{4}{*}{$\begin{array}{c}\text { Clinical } \\
\text { Evaluation }\end{array}$} & 16 & Weight & 3 & $\sqrt{ }$ \\
\hline & & 17 & Height & 2.5 & $\sqrt{ }$ \\
\hline & & 18 & BMIa & 2.8 & $\sqrt{ }$ \\
\hline & & 19 & Function of heart & 3 & $\sqrt{ }$ \\
\hline
\end{tabular}




\begin{tabular}{|c|c|c|c|c|}
\hline & 20 & Blood pressure & 3 & $\sqrt{ }$ \\
\hline & 21 & Respiration & 2.9 & $\sqrt{ }$ \\
\hline & 22 & Asthma & 3 & $\sqrt{ }$ \\
\hline & 23 & Smoking & 3 & $\sqrt{ }$ \\
\hline & 24 & Epileptic seizure & 2.8 & $\sqrt{ }$ \\
\hline & 25 & Sinusitis & 3 & $\sqrt{ }$ \\
\hline & 26 & Foot ulcer & 3 & $\sqrt{ }$ \\
\hline & 27 & Wagner grade & 3 & $\sqrt{ }$ \\
\hline & 28 & Neuropathy & 2.6 & $\sqrt{ }$ \\
\hline & 29 & Ischemic ulcers & 2.8 & $\sqrt{ }$ \\
\hline & 30 & Fever & 1.4 & * \\
\hline & 31 & Diarrhea & 1.1 & * \\
\hline & 32 & Laboratory test (Name of test,Laboratory test results...) & 1.9 & $\sqrt{ }$ \\
\hline & 33 & $\begin{array}{l}\text { Radiography (Name of radiography, radiography } \\
\text { report...) }\end{array}$ & 2 & $\sqrt{ }$ \\
\hline & 34 & Hemoglobin A1c & 2.3 & $\sqrt{ }$ \\
\hline & 35 & HDL b & 3 & $\sqrt{ }$ \\
\hline & 36 & LDL c & 3 & $\sqrt{ }$ \\
\hline & 37 & ACR d & 3 & $\sqrt{ }$ \\
\hline & 38 & $\begin{array}{l}\text { Description of consultation (Consultation type, Consult } \\
\text { orderingPhysician, Consult referring physician, } \\
\text { Comments, diagnosis and advice of referring physician, } \\
\text {..) }\end{array}$ & 2.6 & $\sqrt{ }$ \\
\hline & 39 & Number of HBOT sessions & 2.2 & $\sqrt{ }$ \\
\hline & 40 & Physician order description & 2 & $\sqrt{ }$ \\
\hline & 41 & Nursing observation and assessment & 1.9 & $\sqrt{ }$ \\
\hline & 42 & Date and time Start of HBOT & 3 & $\sqrt{ }$ \\
\hline Procedures & 43 & Vital signs before\&after HBOT(BP,RR,PR) & 2.8 & $\sqrt{ }$ \\
\hline & 44 & HBOT duration (minutes) & 3 & $\sqrt{ }$ \\
\hline & 45 & HBOT pressure (ATA)f & 2.8 & $\sqrt{ }$ \\
\hline & 46 & TcpO2 e after HBOT & 3 & $\sqrt{ }$ \\
\hline & 47 & Time end of HBOT & 3 & $\sqrt{ }$ \\
\hline & 48 & Discharge date & 2.1 & $\sqrt{ }$ \\
\hline Discharge & 49 & Discharge time & 1 & * \\
\hline condition & 50 & Discharge status & 3 & $\sqrt{ }$ \\
\hline & 51 & Medication and educational instructions at discharge & 2.3 & $\sqrt{ }$ \\
\hline & 52 & Description of the follow up order & 2.1 & $\sqrt{ }$ \\
\hline Follow up & 53 & Follow up date & 2 & $\sqrt{ }$ \\
\hline & 54 & Follow up outcome & 2.9 & $\sqrt{ }$ \\
\hline
\end{tabular}

${ }^{a}$ BMI $=$ Body Mass Index $;{ }^{b} \mathrm{HDL}=$ High-density lipoprotein; ${ }^{\mathrm{c}} \mathrm{LDL}=$ Low-density lipoprotein; ${ }^{\mathrm{d}} \mathrm{ACR}=$ Albumin to Creatinine Ratio; ${ }^{\mathrm{e}} \mathrm{TcpO} 2=$ Transcutaneous oxygen pressure, ${ }^{\mathrm{f}} \mathrm{ATA}=\mathrm{Atmospheres}$ Absolute

Table 5. The mean of given scores and selected technical features

\begin{tabular}{|c|c|c|c|c|c|}
\hline Category & Subcategory & No & Data element & Mean & $\sqrt{/} *$ \\
\hline \multirow{11}{*}{ 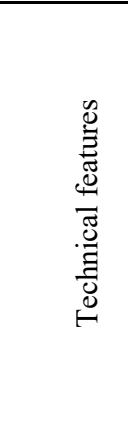 } & \multirow{11}{*}{ N/A } & 1 & Internet access & 2.4 & $\sqrt{ }$ \\
\hline & & 2 & Medication reminder & 2.5 & $\sqrt{ }$ \\
\hline & & 3 & Decision making support & 1.4 & * \\
\hline & & 4 & Provide instructions & 2.8 & $\sqrt{ }$ \\
\hline & & 5 & Being user-friendly & 2.5 & $\sqrt{ }$ \\
\hline & & 6 & Provide HBOT regimens & 2.7 & $\sqrt{ }$ \\
\hline & & 7 & Online scheduling & 3 & $\sqrt{ }$ \\
\hline & & 8 & Providing management dashboard & 2.9 & $\sqrt{ }$ \\
\hline & & 9 & Advanced search & 2.5 & $\sqrt{ }$ \\
\hline & & 10 & Provide report & 3 & $\sqrt{ }$ \\
\hline & & 11 & Register of date, place and causes of death & 2.9 & $\sqrt{ }$ \\
\hline
\end{tabular}




\section{DISCUSSION}

Health care providers need accurate and up-to-date information for their professional activities. In other words, data aggregated in health care information systems should be extensively accessible to all users (Kohli and Tan, 2016). Therefore, improving the quality of information available for health care decision-making is one of the important national efforts that should be made through understanding and implementing appropriate registration systems (Tursunbayeva et al., 2016). The purpose of this study was to determine the requirements of the registry system for HBOT-treated diabetic patients.

The findings of the present study showed that the statistical population selected 25 data elements for administrative category. Most of the data registry systems consist of clinical and administrative groups. The administrative data elements are collected with the aim of identifying and communicating with patients, and including the data necessary for identification, recall and follow up of patients(Khorasani et al., 2019, Lakshminarayanan et al., 2017). Therefore, determining the age and gender of patients, the degree of illness and mortality rate, and identifying the extent of service utilization is required as the basis of management and service delivery (Casalino et al., 2003). As a result, the development of electronic registry systems provides opportunities for improving health services and enhancing applied research. However, providing electronic registries with appropriate functions and standard aggregation of administrative information to support national or global analyses necessities will need the standard requirements (Richesson, 2011).

Based on the findings of the study, 54 data elements were identified for the clinical category, out of which 50 data elements were validated by the statistical population. Standardizing health data collection is an important step in the proper processing of information and getting access to effective health care (Fife et al., 2016). In other words, the crucial factor in the prevention and control of diseases is the availability of accurate and sufficient clinical information about the prognosis, symptoms, type of treatment and the context of each disease occurrence (George et al., 2017, Stoekenbroek et al., 2014). Therefore, gathering diabetes data as a first step in managing diabetes information requires a comprehensive information registry system to record clinical data elements. By analyzing the various indicators obtained from the data recording system, we are able to measure and compare the activities performed for diabetic patients (Richesson, 2011).

The results showed that the experts selected all technical features except the "decision making support". Registry systems provide a set of technical features needed to achieve appropriate clinical results. Planning on these features supports a facility to meet the needs of its environment through the use of registry systems that meet specific health needs. Kawecki et al. in a related study found that technology-based HBOT can heal diabetic foot ulcers, reduce tissue damage, and help reduce the complications of bone and soft tissue infections (Kawecki et al., 2018).

\section{CONCLUSION}

The outcome of the present study was the data elements and technical features of an electronic registry system for HBOT-treated diabetic patients. These requirements make it possible to standardize data collection and improve health care services. Designing a follow-up study to implement and evaluate the electronic registry system for other diseases in HBOT can be a good recommendation for future studies in this field. Clinical data collection using the standard electronic registry system can integrate accurate and detailed data. It is beneficial for all health care providers to provide optimal health services to patients. Standard and accurate clinical data are prerequisites for a convenient access to valid patient information, new treatment modalities and their effects.

\section{ACKNOWLEDGEMENT}

The present study was extracted from the $\mathrm{PhD}$ thesis with code 9321458001 in the field of health information management entitled "Design, development, evaluation an electronic registry system for patients undergoing hyperbaric oxygen therapy" in 2019. We thank the statistical population and all the participants for taking time to contribute to the study. 


\section{REFERENCES}

Ahmadi, M., Mohammadi, A., Chraghbaigi, R., Fathi, T. and Baghini, M.S., 2014. Developing a minimum data set of the information management system for orthopedic injuries in iran. Iranian Red Crescent Medical Journal, Vol. 16, No. 7, pp e17020.

Al-Rubeaan, K.A., Youssef, A.M., Subhani, S.N., Ahmad, N.A., Al-Sharqawi, A.H. and Ibrahim, H.M., 2013. A Web-based interactive diabetes registry for health care management and planning in Saudi Arabia. Journal of medical Internet research, Vol. 15, No. 9, pp e202.

Andersen, N., Hjortdal, J.Ø., Schielke, K.C., Bek, T., Grauslund, J., Laugesen, C.S., Lund-Andersen, H., Cerqueira, C. and Andresen, J., 2016. The Danish registry of diabetic retinopathy. Clinical epidemiology, Vol. 8, No. 1, pp 613-619.

Casalino, L., Gillies, R.R., Shortell, S.M., Schmittdiel, J.A., Bodenheimer, T., Robinson, J.C., Rundall, T., Oswald, N., Schauffler, H. and Wang, M.C., 2003. External incentives, information technology, and organized processes to improve health care quality for patients with chronic diseases. Jama, Vol. 289, No. 4, pp 434-441.

Fife, C.E. and Eckert, K.A., 2018. The Hyperbaric Oxygen Therapy Registry: Driving quality and demonstrating compliance. Undersea \& hyperbaric medicine: journal of the Undersea and Hyperbaric Medical Society, Inc, Vol. 45, No. $1, \mathrm{pp} 1-8$.

Fife, C.E., Gelly, H., Walker, D. and Eckert, K.A., 2016. Rapid analysis of hyperbaric oxygen therapy registry data for reimbursement purposes: Technical communication. Undersea \& hyperbaric medicine: journal of the Undersea and Hyperbaric Medical Society, Inc, Vol. 43, No. 6, pp 633-639.

George, K., Ross, D. and Rowe, L., 2017. Integration of Data to Establish a Standard Operating Procedure for the Diabetic Patient Undergoing Hyperbaric Oxygen Therapy. Journal of Wound Ostomy \& Continence Nursing, Vol. 44, No. 6, pp 546-549.

Hosseini, A., Moghaddasi, H. and Jahanbakhsh, M., 2010. Designing minimum data sets of diabetes mellitus: Basis of effectiveness indicators of diabetes management. Health Information Management, Vol. 7, No. 3, pp 330-340.

Hunt, T. 1972. The effect of varying ambient oxygen tensions on wound metabolism and collagen synthesis. Surg Gynecol Obstet, Vol. 135, No. 1, p 561-567.

Irawan, H., Semadi, I.N. and Widiana, I., 2018. A Pilot Study of Short-Duration Hyperbaric Oxygen Therapy to Improve HbA1c, Leukocyte, and Serum Creatinine in Patients with Diabetic Foot Ulcer Wagner 3-4. The Scientific World Journal, Vol. 16, No. 1.

Kawecki, M., Pasek, J., Cieślar, G., Sieroń, A., Knefel, G., Nowak, M. and Glik, J., 2018. Computerized planimetry evaluation of hyperbaric oxygen therapy in the treatment of diabetic foot. Adv Clin Exp Med, 27(1), pp.39-44.

Khorasani, Z.M., Tara, M., Ebnehoseini, Z., Etminani, K. and Moosavi, Z., 2019. Quantitative Evaluation of Diabetic Medical Record Documentation. Current diabetes reviews, Vol. 15, No. 2, pp 158-163.

Kohli, R. and Tan, S.S.L., 2016. Electronic health records: how can IS researchers contribute to transforming healthcare?. Mis Quarterly, Vol. 40, No. 3, pp 553-573.

Lakshminarayanan, S., Kar, S.S., Gupta, R., Xavier, D. and Reddy, S.V.B., 2017. Primary healthcare-based diabetes registry in Puducherry: Design and methods. Indian journal of endocrinology and metabolism, Vol. 21, No. 3, pp 373-377.

Mathieu, D., Marroni, A. and Kot, J., 2017. Tenth European Consensus Conference on Hyperbaric Medicine: recommendations for accepted and non-accepted clinical indications and practice of hyperbaric oxygen treatment. Diving and hyperbaric medicine, Vol. 47, No. 1, p 24.

Noori, T., Ghazisaeedi, M., Aliabad, G.M., Mehdipour, Y., Mehraeen, E., Conte, R. and Safdari, R., 2019. International comparison of thalassemia registries: Challenges and opportunities. Acta Informatica Medica, Vol. 27, No. 1, p 58.

Richardsson, R. L. 2011. Data standards in diabetes patient registries. Journal of Diabetes Science and Technology, Vol. 5, No. 1, pp 476-485.

Ruthenborg, R.J., Ban, J.J., Wazir, A., Takeda, N. and Kim, J.W., 2014. Regulation of wound healing and fibrosis by hypoxia and hypoxia-inducible factor-1. Molecules and cells, Vol. 37, No. 9, pp 637-643.

Saputri, E.G., Setiani, O. and Dewanti, N.A.Y., 2018. Hubungan riwayat pajanan pestisida dengan kejadian diabetes melitus tipe 2 pada petani penyemprot di kecamatan Ngablak kabupaten Magelang. Jurnal Kesehatan Masyarakat (e-Journal), Vol. 16, No. 1, pp 645-653.

Stoekenbroek, R.M., Santema, T.B., Legemate, D.A., Ubbink, D.T., Van Den Brink, A. and Koelemay, M.J.W., 2014. Hyperbaric oxygen for the treatment of diabetic foot ulcers: a systematic review. European journal of vascular and endovascular surgery, Vol. 47, No. 6, pp 647-655.

Sundh, J., Bornefalk-Hermansson, A., Ahmadi, Z., Blomberg, A., Janson, C., Currow, D.C., McDonald, C.F., McCaffrey, N. and Ekström, M., 2019. REgistry-based randomized controlled trial of treatment and Duration and mortality in long-term OXygen therapy (REDOX) study protocol. BMC pulmonary medicine, Vol. 19, No. 1, pp 50-58.

Tursunbayeva, A., Bunduchi, R., Franco, M. and Pagliari, C., 2017. Human resource information systems in health care: a systematic evidence review. Journal of the American Medical Informatics Association, Vol. 24, No. 3, pp 633-654. 